\title{
Development of a Medication Monitoring System for an Integrated Multidisciplinary Program of Assertive Community Treatment (IMPACT) Team
}

\author{
Sarah C. Watkins*, PharmD Candidate ${ }^{1}$; Bruce R. Winchester*, PharmD Candidate ${ }^{1}$; Nancy C. Brahm, PharmD, MS, BCPP, CGP, \\ Clinical Professor ${ }^{1}$; and Nicole B. Washington, DO, Assistant Professor ${ }^{2}$ \\ ${ }^{1}$ University of Oklahoma College of Pharmacy and ${ }^{2}$ Department of Psychiatry, School of Community Medicine, Tulsa, OK
}

Key words: antidepressant, antipsychotic, assertive community treatment, medication management, monitoring

\begin{abstract}
Purpose: The primary goal was to improve medication management oversight for a severely mentally ill (SMI) community-based population by developing a medication monitoring system based on current guidelines to optimize pharmacotherapy and minimize potential medication-related adverse effects. The secondary goal was improvement in coordination of care between healthcare providers. Methods: Guidelines for medication used for psychiatric indications were reviewed. A database of medication for psychiatric indications with monitoring recommendation was developed. Results: Medication regimens for 68 members of the Integrated Multidisciplinary Program of Assertive Community Treatment (IMPACT) program qualified for review. Fourteen medications, carbamazepine, chlorpromazine, clozapine, fluphenazine and fluphenazine long-acting injections (LAI), haloperidol and haloperidol LAl, lithium, lurasidone, olanzapine, paliperidone and paliperidone LAl, perphenazine, quetiapine, risperidone and risperidone LAI, valproic acid/divalproex, and ziprasidone, were identified. In total, 111 medications are used on a monthly basis. Each member receives more than one medication qualifying for review. Additional monitoring parameters that were evaluated included changes in laboratory orders for members with insulin-dependent diabetes. Annual lipid panels were changed to every 6 months, if applicable. Conclusions and Future Directions: This medication monitoring program was developed to help ensure IMPACT members receive the most effective care and minimize potential medication-related adverse effects. The secondary goal was to improve coordination of care. Medication monitoring will be added as a continuous quality assurance measure. Lab results will be reviewed at least monthly. The medication monitoring program will be evaluated annually.
\end{abstract}

\section{Introduction}

Originally called training in community living ${ }^{1}$, programs of assertive community treatment (PACT) provide an organizational structure for clearly defined service delivery to persons diagnosed with severe mental illnesses (SMI) residing in the community. The emphasis is on a team approach with a multidisciplinary group of mental health professionals providing care for those with a SMI working and residing in a community-based setting. PACT teams have a holistic service delivery approach providing assistance with finances, housing, medical services, and medication management. These services are needed to address the special needs of those with a SMI diagnosis. It has been reported that this population has an increased mortality risk of up to 25 years shorter compared to the general population. ${ }^{2}$ The primary health risk factor in this cohort, particularly those with a diagnosis of schizophrenia, is cardiovascular-related, with special emphasis on elevated lipids. ${ }^{3,4}$ The impact of lifestyle choices, such as diet and exercise, cognitive impairment as a

\section{Corresponding Author:}

Nancy C. Brahm, PharmD, MS, BCPP, CGP, Clinical Professor, College of Pharmacy, University of Oklahoma, 4502 E. $41^{\text {st }}$ Street, 2H17, Tulsa, OK 75135-2512, Phone: 918.660.3579, Fax: 918.660.3009, Email: nancy-brahm@ouhsc.edu consequence of the SMI, and antipsychotic-mediated weight gain can all contribute. Additional variables are the choice of antipsychotic, body mass index at time of medication initiation, and duration of medication use. ${ }^{5}$

Economic barriers pose another challenge. Medication adherence may be a problem. Acquisition and storage are challenges in persons with unstable living arrangements, such as in shelters or homelessness. ${ }^{6}$ PACT teams also address these needs.

We describe the development of a medication monitoring system to help ensure members enrolled in an integrated multidisciplinary program of assertive community treatment (IMPACT) in one institution are evaluated for potential medication-related adverse effects associated with medications used to treat psychiatric illnesses. The secondary goal was to improve coordination of care between the primary care providers (PCPs) and the IMPACT team.

\section{Overview of the Service}

The IMPACT team is a university-based service comprised of a psychiatrist, psychiatry resident, team leader (Licensed Clinical Social Worker), assistant team leader (Licensed Professional Counselor), two case managers, one recovery 
support specialist, three nurses, an administrative assistant, and a clinical pharmacist. Member enrollment is limited to 75 persons. Criteria for enrollment are based on the Diagnostic and Statistical Manual of Mental Disorders, $4^{\text {th }}$ edition, text revision (DSM-IV-TR) ${ }^{7}$ for adults with a primary diagnosis of schizophrenia, bipolar disorder, or other psychotic disorders. Admission criteria are listed in Appendix 1.

IMPACT members are seen for medication management on an individual basis. Depending on the member's ability to function in the community setting and resources available, such as housing, an IMPACT provider may visit from once daily to no less than once weekly. Each member is seen in at least monthly in clinic by the psychiatrist, psychiatry resident, and clinical pharmacist. Prior to this initiative, medication monitoring had not been systematically evaluated through database management.

\section{Database Development}

Creation of a medication management database for psychotropic monitoring was undertaken. The literature was reviewed for evidence-based guidelines for monitoring of the following categories: antipsychotics, antidepressants, and mood stabilizers. Search terms included antidepressant, antipsychotic, bipolar disorder, guidelines, medication management, mood stabilizer, schizoaffective, and schizophrenia. Databases used for searches were EBSCOhost, Medline, PsycINfo, and PubMed for the period 1968 to March 2012. After the literature was obtained, at least two of the authors reviewed each article.

This project was reviewed and approved by the institutional review board. Anonymity was maintained throughout the project. Inclusion of information into the IMPACT database was protected in accordance with university policies and procedures. Individual monitoring information was categorical and for analysis only. No protected health information was involved with aggregate reporting. Descriptive statistics were used.

\section{Monitoring Needs and Results}

Medication regimens for 68 IMPACT members qualified for review. Fourteen medications, including products available as both long-acting injection (LAI) and oral formulations, were identified for inclusion in the database for monitoring. A total of 111 medications, including four LAls, were found to be used on a monthly basis. Each IMPACT member had more than one medication entered into the medication management database.

Once the database was developed and all medication regimens entered, the medical director reviewed the charts to determine if additional monitoring was needed on a caseby-case basis. Additional changes were made. Orders for fasting blood glucose were discontinued $(n=2,100 \%)$ and changed [to 'attempt fasting status' and 'obtain glycosylated hemoglobin (A1C)] and scheduled for every 6 months. Annual lipid panels were changed to every 6 months.

\section{Discussion}

PACT teams are not new. The first initiatives were reported in the $1970 \mathrm{~s}^{1}{ }^{1}$ The focus at that time was housing and substance abuse treatment. ${ }^{8}$ The role of the pharmacist providing clinical services to chronic psychiatric outpatients was reported in $1978 .{ }^{9}$ In addition to that initial work, the role of the pharmacist in mental health settings has been recognized and the advantages evaluated. ${ }^{10}$ One example was the development of a psychiatric pharmacy clinic for indigent patients. In this initiative, an underserved population of clinic patients with mental health concerns was referred to the psychiatric pharmacist. Outcomes included a greater than $90 \%$ acceptance rate of pharmacist-initiated recommendations and cost savings. ${ }^{11}$

\section{Why Scheduled Monitoring Is Needed}

Persons with SMIs face a number of challenges. These include (but are not limited to) comorbidities of substance use disorders, homelessness, chronic health conditions, and metabolic and/or endocrine disorders secondary to medication use. The IMPACT program includes scheduled monitoring for movement disorders and medication reviews. Specific medications are monitored as identified in Appendix 2.

In addition to medication-related adverse effects the patient may experience and may or may not report, monitoring for endocrine and metabolic changes has the potential to avoid long-term medication-induced consequences. Life expectancy for persons with a diagnosis of schizophrenia has been correlated with increased mortality equating with a lifespan up to 25 years shorter. ${ }^{2}$ In a meta-analysis involving 152 mortality studies, the risk of premature death in persons with a diagnosis of schizophrenia was twice as great when compared to persons in the general population. Cardiovascular diseases were found to be the leading mortality event. Risk factors included obesity, hypertension, dyslipidemia, and elevated glucose levels. The authors recognized the predisposition to adverse metabolic consequences did include contributing factors amenable to change, such as dietary choices and exercise. Factors associated with significant antipsychotic-induced weight gain were a lower body mass index at pharmacotherapy initiation, choice of agent, duration of use, and age (younger vs. older patients). The choice of first or second generation agent had 
an effect on lipid changes. Clozapine, olanzapine, and quetiapine were associated with more hyperlipidemia. Haloperidol was less associated with lipid elevations than chlorpromazine for first generation agents. Consistent with recommendations for the general population, the author recommended regular exercise, weight management (or weight loss), blood pressure control, and smoking cessation as management strategies. Medication monitoring recommendations included baseline glucose evaluations with monitoring no less than every 6 months.

The presence of diabetes and schizophrenia may be associated with poorer glucose control compared to persons not dually diagnosed with diabetes and a SMI (schizophrenia specifically) although results differed with the study populations. ${ }^{12,13}$ A number of strategies were proposed for prolactin elevations, but specific monitoring guidelines were not provided. ${ }^{5}$ The medication monitoring form developed for use in the IMPACT program includes general patient information (weight or body mass index, waist circumference, and vital signs), a lipid panel, laboratory work specific for mood stabilizers (lithium, divalproex, and carbamazepine), and an assessment for movement disorders (Abnormal Involuntary Movement Scale or AIMS).

One of the anticipated outcomes with the creation of the database was coordination of care with the primary care provider (PCP). Prior to the database, the comprehensive metabolic panel and complete blood count were ordered by the PCP. Records and appointments with the PCP were not always available to the IMPACT team. Some providers have electronic data management; others do not. A specific example of why coordination of care and systematic lab review is needed is provided.

\section{Case Example}

Psychiatric medication-related monitoring was ordered and managed by the psychiatrist with correspondence of the results transmitted to the PCP via an electronic medical record (EMR). Posted results from outside providers, like labs or diagnostic tests, could take up to $\mathbf{3 0}$ days to be entered into the system. Lab results were not readily available for this patient. This resulted in the need for a medical hospitalization for a 34-year old IMPACT member (white male) with medical comorbidities of high density lipoproteins (HDL) less than 40 $\mathrm{mg} / \mathrm{dL}$ (desired range for men: $>40 \mathrm{mg} / \mathrm{dL}$ ), hypertriglyceridemia, tachycardia, and obesity. Psychiatric diagnoses of schizophrenia, paranoid type, anxiety not otherwise specified, and nicotine dependence were wellcontrolled with a regimen of clozapine $800 \mathrm{mg}$, divalproex extended release $2000 \mathrm{mg}$, zolpidem $10 \mathrm{mg}$, and propranolol extended release $160 \mathrm{mg}$, all administered at bedtime.
Medication prescribed by the PCP had been discontinued as the member had not been seen by the provider for approximately 18 months. This information was not provided to the IMPACT team. The member received home-based services from the team three times weekly. Four days prior to hospitalization the member was seen by the IMPACT nurse. He complained of flu-like symptoms and flank pain. He declined any interventions, including medical assistance. One day prior to admission, he was again seen by the nurse. The member declined to contact his PCP or schedule a visit. The following day the member was admitted for medical reasons. A diagnosis of pancreatitis was made at that time. Diabetes was diagnosed during this inpatient admission. Had a system for scheduled, systematic medication monitoring and coordination of care been in place, we theorize that hospitalization may have been avoided with better communication between healthcare providers.

\section{Monitoring as a Quality Assurance Activity}

While pharmacy-based interventions in medication adherence $^{14}$ and clinical pharmacist interventions with a PACT team ${ }^{15}$ have been previously reported, programs with regular pharmacist involvement in longitudinal antipsychotic monitoring were not found. The goals of the monitoring program were to help ensure members receive the most effective care, to minimize potential medication-related adverse effects and to improve coordination of care between medical and psychiatric providers. It is anticipated this monitoring program will address these goals.

In addition, the monitoring program was incorporated into the continuous quality assurance activities used by the IMPACT team and represents an ongoing activity. Members are scheduled for clinic either twice a month or monthly. Lab results are reviewed and discussed at least monthly during clinic appointments. The program will be evaluated annually and adjusted as needed to meet the needs of the IMPACT members.

Individual member monitoring also was incorporated into the monthly medication reviews done by the psychiatric resident and reviewed by the psychiatrist and clinical pharmacist. The form and compete information have been added to the service-specific university-maintained secure drive.

(Appendix 2)

\section{Challenges and Future Directions}

Ongoing challenges for coordination of care for a psychiatrically and medically complex population include ensuring medication regimens are reviewed and the reviewer has access to the most current information. Developing the database and including this information on a shared service- 
specific drive were implemented to facilitate integration. Programs interested in adding database monitoring development need to provide resources for this task. We found this process involved significant time for development and revisions. Resources available for development included access to an electronic medical record (EMR). This also was one of the limitations: access to an EMR. Identifying the group of service providers that utilize an EMR and those that do not and addressing coordination of care with the needs of each provider group will help ensure better medication management between medical and psychiatric areas of concern.

Because the medication monitoring database has been in existence for only a short time, long-term gains cannot be reported at this time. A more comprehensive medication management project is in development to specifically identify pharmacist-managed interventions and how these interventions benefitted the IMPACT member, service, and program. Specific areas include clearly identifying interventions and outcomes. It is not known at this time if cost savings will be included as a project outcome. In addition, implementation methods are in development to address the secondary goal of improving coordination of care with identification of resources for this phase. Following implementation of this component, it is anticipated one year of data collection will help identify and focus what additional oversight is needed in this area. Inclusion of primary medication concerns will be evaluated for inclusion.

\section{Conclusions}

Multiple medications to treat patients with severe mental illness are used on a regular basis for members enrolled in the IMPACT program to address their psychiatric needs. Development of a database to facilitate psychiatricallyindicated medication monitoring, help ensure members enrolled in the IMPACT program, and improve coordination of care between PCPS and the IMPACT team was undertaken. Additional phases to address these goals will be needed. Integration into scheduled monitoring in a readily available format will be reviewed to ensure the information is updated and follow-up initiated.

\section{References}

1. Test MA, Stein LI. Training in community living: a follow-up look at a gold-award program. Hosp Community Psychiatry 1976;27:193-194.

2. Laursen TM, Munk-Olsen T, Vestergaard M. Life expectancy and cardiovascular mortality in persons with schizophrenia. Curr Opin Psychiatry 2012;25:8388.
3. Newcomer JW, Hennekens $\mathrm{CH}$. Severe mental illness and risk of cardiovascular disease. JAMA 2007;298:1794-1796.

4. Osby U, Correia N, Brandt L, et al. Mortality and causes of death in schizophrenia in Stockholm county, Sweden. Schizophr Res 2000;45:21-28.

5. Monteleone $\mathrm{P}$, Martiadis V, Maj M. Management of schizophrenia with obesity, metabolic, and endocrinological disorders. Psychiatr Clin North Am 2009;32:775-794.

6. Muir-Cochrane E, Fereday J, Jureidini J, et al. Selfmanagement of medication for mental health problems by homeless young people. Int J Ment Health Nurs 2006;15:163-170.

7. American Psychiatric Association. Diagnostic and Statistical Manual of Mental Disorders, 4th ed., Text Revision. Washington, DC, American Psychiatric Association, 2000.

8. Meisler N, Blankertz L, Santos AB, McKay C. Impact of assertive community treatment on homeless persons with co-occurring severe psychiatric and substance use disorders. Community Ment Health 1997;33:113-122.

9. Rosen CE, Copp WM, Holmes S. Effectiveness of a specially trained pharmacist in a rural community mental health center. Public Health Rep 1978;93:464-467.

10. Finley PR, Crismon ML, Rush AJ. Evaluating the impact of pharmacists in mental health: a systematic review. Pharmacotherapy 2003;23:1634-1644.

11. Caballero J, Souffrant G, Heffernan E. Development and outcomes of a psychiatric pharmacy clinic for indigent patients. Am J Health Syst Pharm 2008;65:229-233.

12. Dixon LB, Kreyenbuhl JA, Dickerson FB, et al. A comparison of type 2 diabetes outcomes among persons with and without severe mental illnesses. Psychiatr Serv 2004;55:892-900.

13. Ogawa M, Miyamoto Y, Kawakami N. Factors associated with glycemic control and diabetes selfcare among outpatients with schizophrenia and type 2 diabetes. Arch Psychiatr Nurs 2011;25:63-73.

14. Valenstein $M$, Kavanagh J, Lee $T$, et al. Using a pharmacy-based intervention to improve antipsychotic adherence among patients with serious mental illness. Schizophr Bull 2011;37:727736.

15. Gable KN, Stunson MJ. Clinical pharmacist interventions on an assertive community treatment team. Community Ment Health 2010;46:351-355. 
This work was presented as a student poster at the $15^{\text {th }}$ Annual Meeting of the College of Psychiatric and Neurologic Pharmacists (CPNP), April 29 - 30, 2012, Tampa, FL.

Financial Disclosure: No similar work by the authors is under review or in press. No funding was requested or received in conjunction with this manuscript. The authors report no known or suspected conflicts of interest related, but not limited, to consulting fees, paid expert testimony, employment, grants, honoraria, patents, royalties, stocks, or other financial or material gain involve with or pertaining to the subject matter of this manuscript.

*Updated Author Information: At the time this work was developed, Sarah C. Watkins and Bruce R. Winchester were PharmD Candidates at The University of Oklahoma College of Pharmacy. Both Dr. Watkins and Winchester are in residency programs. Current information is provided:

Sarah C. Watkins, PharmD, LT, USPHS

PGY1 Pharmacy Practice Resident

Cherokee Nation W.W. Hastings Hospital

100 South Bliss Avenue, Tahlequah, OK 74464
Bruce Winchester, PharmD

PGY1 Pharmacy Practice Resident

Saint Francis Hospital

6161 South Yale Avenue, Tulsa, OK 74136 


\section{Appendix 1}

Admission Criteria for the IMPACT Program

Primary Criterion:

- Adults with a primary diagnosis of schizophrenia or other psychotic disorders limited to schizoaffective disorder or bipolar disorder with psychotic features.

Additional criteria of at least four of the following risk factors:

- Admission and/or length-of-stay (LOS) parameters for psychiatrically-related admissions: four hospitalizations OR a LOS greater than 30 days total time in the past 12 months;

- Severe symptoms that are persistent or recurrent and are affective, psychotic, or suicidal;

- A comorbid substance abuse disorder in existence more than 6 months;

- Either high risk for or involvement in the criminal justice system in the past 12 months;

- If not homeless, there is an imminent risk for homelessness OR housing is unsafe or substandard; and/or

- Functional status has been clinically evaluated such that supported housing as a residential choice is possible if intensive services are provided OR supported housing is required if more intensive services are not available. 
Appendix 2

IMPACT Antipsychotic Monitoring

\begin{tabular}{|l|l|l|l|l|l|}
\hline Monitor Needed/Date & Baseline & $\mathbf{1 2}$ Weeks & Monthly & Every 6 months & Annually \\
\hline CMP & & & & & \\
\hline Weight/BMI & & & & & \\
\hline Waist circumference & & & & & \\
\hline BP & & & & & \\
\hline Fasting BG/A1c & & & & & \\
\hline Fasting Lipid Profile & & & & \\
\hline
\end{tabular}

$\mathrm{CMP}=$ comprehensive metabolic panel; $\mathrm{BMI}$ = body mass index; $\mathrm{BP}=$ blood pressure; $\mathrm{BG}$ = blood glucose

Current BMI: $\mathrm{Ht}:$ Weight:

Fasting Lipid Panel

Date of most recent labs:

Results: Cholesterol:

Triglycerides:

LDL:

HDL:

Ordered:

$\mathrm{LDL}=$ low-density lipoprotein; $\mathrm{HDL}=$ high-density lipoprotein

Comments:

Fasting Blood Glucose (FBG) or Glycosylated hemoglobin (A1c)

Date of most recent lab(s):

Results: FBG:

A1c:

Ordered:

Comments:

Lithium (Li): every 6 months

Date of most recent lab(s):

Results: BMP:

TSH:
Li Level:
Ordered:

$\mathrm{BMP}=$ basic metabolic panel; $\mathrm{TSH}=$ thyroid stimulating hormone

Comments:

Valproic Acid (VPA): every 6 months

Date of most recent lab(s):

Results: CMP:

$$
\begin{aligned}
& \text { CBC: } \\
& \text { VPA Level: } \\
& \text { Ordered: }
\end{aligned}
$$

$\mathrm{CMP}$ = comprehensive metabolic panel; $\mathrm{CBC}=$ complete blood count

Comments:

Carbamazepine (CBZ): every 6 months

Date of most recent lab(s):

Results: CMP:

CBC:

CBZ Level:

Ordered:

Comments:

Abnormal Involuntary Movement Scale (AIMS): Score/date:

1 